\title{
Inclusão social do deficiente visual - experiência e resultados de Assis
}

\author{
Social inclusion for visually impaired - experience and results from Assis
}

\author{
Eduardo Andreghetti ${ }^{1}$ \\ Maria Rosa Bet de Moraes Silva ${ }^{2}$ \\ Newton Kara José ${ }^{3}$ \\ Maurício Maia ${ }^{4}$ \\ Mariana de Rezende Andreghetti $\mathrm{i}^{5}$ \\ Valcir Coronado Antunes ${ }^{6}$
}

Trabalho realizado no Departamento de Oftalmologia da Universidade Estadual Paulista - UNESP - São Paulo (SP) - Brasil.

Mestre em Bases Gerais da Cirurgia, Faculdade de Medicina de Botucatu - Universidade Estadual Paulista UNESP - Botucatu (SP) - Brasil.

Professora Livre-Docente do Departamento de Oftalmologia, Otorrinolaringologia e Cirurgia de Cabeça e Pescoço da Faculdade de Medicina de Botucatu - UNESP Botucatu (SP) - Brasil.

${ }^{3}$ Professor Titular de Oftalmologia da Universidade Estadual de Campinas - UNICAMP - Campinas (SP) - Brasil.

${ }^{4}$ Orientador da Pós-Graduação do Departamento de Oftalmologia da Universidade Federal de São Paulo UNIFESP - São Paulo (SP) - Brasil.

${ }^{5}$ Estudante do $6^{\circ}$ ano de Medicina - Universidade de Santo Amaro - UNISA - São Paulo (SP) - Brasil.

${ }^{6}$ Médico Oftalmologista do Instituto de Oftalmologia de Assis - Assis (SP) - Brasil.

Endereço para correspondência: Maria Rosa Bet de Moraes Silva. Faculdade de Medicina de Botucatu UNESP - Depto. OFT/ORL/CCP - Distrito de Rubião Júnior s/n - Botucatu (SP) CEP 18618-970

E-mail: rosabet@fmb.unesp.br

Recebido para publicação em 29.10.2007

Última versão recebida em 06.10.2009

Aprovação em 07.10.2009

\section{RESUMO}

Objetivos: Traçar o perfil do usuário da Sala de Recursos para Deficiente Visual na cidade de Assis - SP, avaliar a porcentagem de deficientes visuais empregados e comparar o emprego com idade, gênero, tipo de deficiência e doença causadora da deficiência, entre dois períodos: de 1984 a 1996 e de 1997 a 2009. Métodos: Foi realizado estudo retrospectivo dos prontuários médicos e da ficha escolar dos portadores de deficiência visual que frequentaram a Sala de Recursos para Deficiente Visual, no período de 1984 a 2009 na cidade de Assis - SP, divididos em dois períodos de 1984 a 1996 (G1) e de 1997 a 2009 (G2). Analisaram-se dados demográficos, a doença que provocou a baixa visão, o tipo de deficiência visual (cegueira ou baixa visão), escolaridade, recursos ópticos, frequência à Sala de Recursos para Deficiente Visual e taxa de emprego. Foi feita associação da taxa de emprego com: idade, gênero, raça/cor, tipo de deficiência e doença nos dois diferentes períodos. Resultados: Foram encontrados 149 deficientes visuais sendo: $61,07 \%$ homens, $38,9 \%$ mulheres, $82,5 \%$ brancos e $17,4 \%$ não brancos e a mediana da idade foi de 18 anos. Dos 149 deficientes visuais, $63,75 \%$ eram portadores de baixa visão e $36,24 \%$ portadores de cegueira. As principais doenças que levaram à deficiência visual dos 149 pacientes foram em ordem decrescente: retinocoroidite por toxoplasmose $(17,40 \%)$, atrofia óptica congênita $(12,10 \%)$, alta hipermetropia $(8,72 \%)$, retinose pigmentar e alta miopia $(6,71 \%$ cada uma) e glaucoma congênito e catarata congênita $(6,04 \%$ cada uma). A frequência à Sala de Recursos para Deficiente Visual foi boa em mais de $50 \%$ dos pacientes. Estavam trabalhando regularmente $44,7 \%$ e $12,3 \%$ dos maiores de 14 anos respectivamente nos períodos de 1984 - 1996 e de 1997 - 2009. Não houve diferença entre os dois períodos quanto às características demográficas, tipo de deficiência e doenças, havendo correlação da taxa de emprego apenas com a idade (pacientes com média de idade maior apresentavam maior porcentagem de emprego). Conclusões: Os deficientes visuais eram na maioria homens, brancos, portadores de baixa visão e portadores de toxoplasmose ocular. Quase metade $(44,7 \%)$ dos pacientes com mais de 14 anos encontrava-se trabalhando regularmente até 1996 sugerindo que esforços conjuntos de oftalmologistas e educadores auxilia na inclusão social destes pacientes. A taxa de emprego caiu no período de 1997 a 2009 e a de aposentados aumentou.

Descritores: Transtornos da visão; Cegueira; Baixa visão/reabilitação; Emprego; Serviços de saúde comunitária; Socialização 


\section{INTRODUÇ̃̃O}

Desde o cego congênito até aquele que perdeu a visão por doenças progressivas ou acidentes, tem-se que adequar as forças comunitárias a fim de que se proceda a um desenvolvimento neuro-psico-físico, reabilitação e educação adequados para propiciar sua inclusão social.

$\mathrm{O}$ acesso dos deficientes visuais a um "centro de referência" e sua aderência às possibilidades ali oferecidas devem ser uma preocupação constante daqueles envolvidos nos cuidados a esses pacientes, criando mecanismos que inspirem confiança aos deficientes, por meio de propostas que possam integrá-los à sociedade, proporcionando-lhes facilidades como transporte, alimentação, materiais didáticos apropriados e outros que minimizem suas grandes dificuldades naturais ${ }^{(1)}$.

Os médicos oftalmologistas têm importância fundamental nesse processo, uma vez que com sua atuação profissional, podem avaliar o grau de deficiência do paciente, prescrever tratamentos e recursos ópticos especiais, além de poderem mobilizar a sociedade para que seja formada uma equipe permanente de ajuda a esses deficientes visuais.

Em 1983, a cidade de Assis, sudoeste do Estado de São Paulo (hoje com 92.000 habitantes) não contava com nenhuma instituição organizada de educação para deficientes visuais (portadores de baixa visão e cegos).

Em 1984, deu-se início à Sala de Recursos para Deficiente Visual (SRDV) ${ }^{(2-3)}$ em uma escola estadual da cidade de Assis e os deficientes visuais passaram a frequentá-la logo após a avaliação e prescrição oftalmológicas realizadas por oftalmologistas voluntários. Os oftalmologistas lideraram movimento da sociedade para que os deficientes visuais pudessem ter acesso não só aos auxílios prescritos mas também atendimento multidisciplinar com psicólogos e pedagogos. As atividades da SRDV e os atendimentos oftalmológicos e multidisciplinar continuaram até a presente data.

\section{OBJETIVOS}

Traçar o perfil do usuário da SRDV na cidade de Assis - SP, avaliar a porcentagem e perfil dos deficientes visuais empregados e correlacionar o emprego com idade, gênero, tipo de deficiência e doença entre dois períodos de 1984 a 1996 e de 1997 a 2009.

\section{MÉTODOS}

Foi realizado estudo retrospectivo dos prontuários médicos e da ficha escolar dos deficientes visuais que frequentaram a SRDV no período de 1984 a 2009 na cidade de Assis - SP. Os deficientes visuais foram divididos em dois grupos: grupo 1 (G1) formado pelos deficientes que iniciaram as atividades na SRDV de 1984 a 1996 e grupo 2 (G2) por aqueles que iniciaram as atividades de 1997 a 2009 (após o Decreto n 3.298 que regulamentou a Lei 7.857 que concede benefícios e previdência social aos deficientes).
Todos os deficientes visuais foram submetidos a exame e acompanhamento oftalmológico e psicológico além das atividades na SRDV.

Antes de frequentarem a SRDV os pacientes foram submetidos ao exame oftalmológico completo que constou de: acuidade visual (AV) para longe e para perto e com tabela logarítmica, refração estática e dinâmica, fundo de olho, biomicroscopia, tonometria, campimetria e teste de visão a cores e quando necessário ultrassonografia, ecobiometria e angiofluoresceinografia. Após o exame oftalmológico os pacientes foram classificados em cegos $\left(\mathrm{AV}<20 / 400\right.$ e/ou campo visual entre 5 e $\left.10^{\circ}\right)$ ou portadores de baixa visão ( $A V \geq 20 / 400$ até $\leq 20 / 70)$ e foram analisadas as doenças causadoras da deficiência visual. Foram receitados recursos ópticos e não ópticos baseados nas necessidades individuais.

Uma vez classificados quanto à deficiência visual e após receber os recursos ópticos e não ópticos (por doação da sociedade), os pacientes foram encaminhados à SRDV e continuaram também a frequentar a escola normal correspondente ao seu grau de escolaridade. Os pacientes foram separados por escolaridade em: Jardim, Primeiro Grau, Segundo Grau e Universitário. Aqueles que não puderam ser matriculados nas classes mencionadas, mas frequentaram a SRDV, foram chamados de "Sem registro" (SR) e os que apresentavam além da deficiência visual, deficiência mental, foram também encaminhados para uma "Classe especial" onde eram orientados por pedagoga especializada em deficiência mental. Os pacientes adultos, em geral já alfabetizados, foram encaminhados apenas à SRDV. O comparecimento à SRDV foi considerado como fraco quando de 1 a 3 anos, bom de 4 a 9 anos e ótimo de 10 a 12 anos. Chamouse "evadido" o paciente que abandonou a SRDV.

Foram analisados: características demográficas, causas da deficiência visual, tipo de deficiência, recursos ópticos, escolaridade, frequência à SRDV e taxa de emprego (dos acima de 14 anos - idade mínima para o trabalho requerida pela CLT) ${ }^{(4)}$ em ambos os grupos.

Comparou-se também a associação da taxa de emprego com: idade, gênero, raça/cor, tipo de deficiência e doença entre os dois grupos.

Para análise estatística foi usado o programa SPSS versão 15. As associações foram estimadas utilizando teste exato de Fisher. A comparação entre empregados e não empregados em relação às variáveis numéricas observadas foi feita pelo teste de Mann-Witney.

Este estudo teve aprovação do Comitê de Ética em Pesquisa em Seres Humanos do Hospital Regional de Assis (Parecer $n^{\circ}$ 65/2007).

\section{RESULTADOS}

\section{Características demográficas}

Iniciaram as atividades na SRDV, 149 pacientes 58 no período de 1984 a 1996 (G1) e 91 no período de 1997 a 2009 (G2). Dos $149,82,5 \%$ eram brancos e $17,4 \%$ não brancos e $61,07 \%$ 
eram homens e 38,9\% mulheres. A mediana da idade foi de 18 anos (mínima de 1 e máxima de 69 anos) e quanto à distribuição etária 7,4\% estavam entre 4 e 9 anos, 22,14\% entre 9 e 14 anos, $21,5 \%$ entre 14 e 19 anos, $13,42 \%$ entre 19 e 24 anos e $34,23 \%$ tinham 24 anos ou mais. Nos 2 grupos a maioria dos pacientes se encontrava nas faixas etárias de 9 a 24 anos (Tabela 1).

\section{Escolaridade}

Em relação à escolaridade no G1: 5,2\% frequentavam o Jardim, $50 \%$ o Primeiro grau, $8,6 \%$ o Segundo grau, 3,4\% a Universidade e $32,7 \%$ eram deficientes mentais, "sem registro" ou "evadidos" e no G2: 3,3\% frequentavam o Jardim, 37,4\% o Primeiro grau, $35,2 \%$ o Segundo grau, $5,5 \%$ a Universidade e $35,3 \%$ eram deficientes mentais, "sem registro" ou "evadidos". Houve diferença significativa ( $\mathrm{p}=0,001)$ quanto à escolaridade entre os dois grupos sendo maior no G2, mas não houve diferença entre os pacientes empregados, $\mathrm{p}=0,084$ (Tabela 2).

\section{Frequência à sala de recursos}

No G1 tiveram frequência considerada fraca $37,9 \%$ dos pacientes, boa $56,9 \%$ e ótima $5,2 \%$ pacientes e no G2: fraca $38,5 \%$, boa $51,6 \%$ e ótima $9,9 \%$. Não houve diferença entre os 2 grupos quanto a frequência à SRDV (Tabela 2).

\section{Recursos ópticos}

Receberam pelo menos um recurso óptico $74,1 \%$ dos pacientes em G1 e 63,7\% em G2 (Tabela 2).

\section{Tipo de deficiência}

Quanto ao tipo de deficiência visual no G1: 29,3\% eram cegos e $70,7 \%$ portadores de baixa visão e no $\mathrm{G} 2: 40,7 \%$ eram cegos e $59,3 \%$ portadores de baixa visão não havendo diferença entre todos os pacientes dos dois grupos $(\mathrm{p}=0,218)$ e também entre os pacientes empregados dos dois grupos $(\mathrm{p}=0,986)$ (Tabela 3$)$.

\section{Doenças}

As doenças causadoras da deficiência visual dos 149 pacientes foram: em ordem decrescente $17,40 \%$ toxoplasmose ocular, $12,1 \%$ atrofia óptica congênita, 9,39\% glaucoma congênito e catarata congênita $6,04 \%$, retinose pigmentar e alta miopia $6,71 \%$ e alta hipermetropia $8,72 \%$.

Analisando as causas separadamente nos dois grupos foi observado que a toxoplasmose ocular foi a principal causa em ambos com $24,1 \%$ e $19,8 \%$ em G1 e G2 respectivamente mas não nos pacientes empregados. A segunda maior causa foi a atrofia óptica congênita com $17,3 \%$ e 10,9\% respectivamente para G1 e G2. A frequência das demais doenças foi diferente entre os dois grupos e está descrita na tabela 3.

\section{Características $e$ análise dos pacientes empregados}

Estavam empregados 17 pacientes em G1 e 8 pacientes em G2 o que representa respectivamente $44,7 \%$ e $12,3 \%$ dos maiores de 14 anos em ambos os grupos. Não houve diferença para gênero, idade, raça e tipo de deficiência visual entre os dois grupos em relação aos pacientes empregados, mas a mediana da idade foi maior nos pacientes empregados do que nos não empregados.

Não houve também diferença entre as cinco principais causas da deficiência visual em os pacientes empregados de ambos os grupos $(\mathrm{p}=0,119)$.

\section{DISCUSSÃO}

Para inclusão social do deficiente visual é necessário um trabalho multidisciplinar conjunto especialmente de oftalmo-

Tabela 1. Características demográficas dos deficientes visuais que frequentaram a SRDV e dos deficientes visuais empregados (Assis - SP, 1984 - 2009)

\begin{tabular}{|c|c|c|c|c|c|c|c|c|c|}
\hline & & \multicolumn{4}{|c|}{ Amostra total } & \multicolumn{4}{|c|}{ Pacientes empregados } \\
\hline & & \multicolumn{2}{|c|}{ G1 } & \multicolumn{2}{|c|}{ G2 } & \multicolumn{2}{|c|}{ G1 } & \multicolumn{2}{|c|}{ G2 } \\
\hline & & $\mathbf{n}$ & $(\%)$ & $\bar{n}$ & $(\%)$ & $\mathbf{n}$ & $(\%)$ & $n$ & $(\%)$ \\
\hline \multirow{3}{*}{ Gênero } & Feminino & 21 & 36,2 & 37 & 40,65 & 7 & 41,2 & 6 & 75,0 \\
\hline & Masculino & 37 & 63,8 & 54 & 59,34 & 10 & 58,8 & 2 & 25,0 \\
\hline & TOTAL & 58 & 100,0 & 91 & 100,00 & 17 & 100,0 & 8 & 100,0 \\
\hline \multirow[t]{3}{*}{ Raça/cor } & Branca & 49 & 84,5 & 74 & 81,31 & 13 & 76,5 & 5 & 62,5 \\
\hline & Não branca & 9 & 15,5 & 17 & 18,68 & 4 & 23,5 & 3 & 37,5 \\
\hline & TOTAL & 58 & 100,0 & 91 & 100,00 & 17 & 100,0 & 8 & 100,0 \\
\hline \multirow{8}{*}{$\begin{array}{l}\text { Idade } \\
\text { (anos) }\end{array}$} & $<4$ & 0 & 0 & 2 & 2,20 & - & - & - & - \\
\hline & $4-9$ & 9 & 15,5 & 2 & 2,20 & - & - & - & - \\
\hline & $9-14$ & 11 & 19,0 & 22 & 24,20 & - & - & - & - \\
\hline & $14-19$ & 14 & 24,0 & 18 & 19,80 & 3 & 17,7 & 1 & 12,5 \\
\hline & $19-24$ & 9 & 15,5 & 11 & 12,10 & 4 & 23,5 & 0 & 0 \\
\hline & $\geq 24$ & 15 & 26,0 & 36 & 39,60 & 10 & 58,8 & 7 & 87,5 \\
\hline & TOTAL & 58 & 100,0 & 91 & 100,00 & 17 & 100,0 & 8 & 100,0 \\
\hline & & \multicolumn{4}{|c|}{$\mathrm{p}=0,0250^{*}$} & \multicolumn{2}{|c|}{$p=0,0463^{*}$} & & \\
\hline
\end{tabular}




\begin{tabular}{|c|c|c|c|c|c|c|c|c|c|}
\hline & & \multicolumn{4}{|c|}{ Amostra total } & \multicolumn{4}{|c|}{ Pacientes empregados } \\
\hline & & \multicolumn{2}{|c|}{ G1 } & \multicolumn{2}{|c|}{ G2 } & \multicolumn{2}{|c|}{ G1 } & \multicolumn{2}{|c|}{ G2 } \\
\hline & & $\mathbf{n}$ & $(\%)$ & $n$ & $(\%)$ & $\mathrm{n}$ & $\overline{(\%)}$ & $\bar{n}$ & $(\%)$ \\
\hline \multirow[t]{7}{*}{ Escolaridade } & Jardim & 3 & 5,2 & 3 & 3,3 & 0 & 0 & 0 & 0 \\
\hline & $1^{\circ}$ grau & 29 & 50,0 & 34 & 37,4 & 5 & 29,4 & 1 & 12,5 \\
\hline & $2^{\circ}$ grau & 5 & 8,6 & 32 & 35,2 & 4 & 23,5 & 5 & 62,5 \\
\hline & Universitário & 2 & 3,4 & 5 & 5,5 & 2 & 11,7 & 2 & 25,0 \\
\hline & Outros & 19 & 32,7 & 17 & 18,7 & 6 & 35,3 & 0 & 0 \\
\hline & Total & 58 & 100,0 & 91 & 100,0 & 17 & 100,0 & 8 & 100,0 \\
\hline & & \multicolumn{4}{|c|}{$p=0,001^{*}$} & \multicolumn{4}{|c|}{$p=0,084^{*}$} \\
\hline \multirow[t]{5}{*}{ Frequência à SRDV } & Fraca & 22 & 37,9 & 35 & 38,5 & 4 & 23,5 & 4 & 50,0 \\
\hline & Boa & 33 & 56,9 & 47 & 51,6 & 13 & 76,5 & 4 & 50,0 \\
\hline & Ótima & 3 & 5,2 & 9 & 9,9 & 0 & 0 & 0 & 0 \\
\hline & Total & 58 & 100,0 & 91 & 100,0 & 17 & 100,0 & 8 & 100,0 \\
\hline & & \multicolumn{4}{|c|}{$p=0,581^{*}$} & \multicolumn{4}{|c|}{$p=0,359^{*}$} \\
\hline \multirow[t]{4}{*}{ Recursos ópticos } & Com & 43 & 74,1 & 58 & 63,7 & 11 & 64,7 & 5 & 62,5 \\
\hline & Sem & 12 & 20,7 & 31 & 34,1 & 6 & 35,3 & 3 & 37,5 \\
\hline & $<$ que 3 anos & 3 & 5,2 & 2 & 2,2 & 0 & 0 & 0 & 0 \\
\hline & Total & 58 & 100,0 & 91 & 100,0 & 17 & 100,0 & 8 & 100,0 \\
\hline
\end{tabular}

logistas e de educadores. A sociedade e, em particular, os oftalmologistas brasileiros ainda não estão totalmente conscientes de suas respectivas responsabilidades como a promoção e inclusão dos deficientes visuais. Infelizmente, a grande maioria dos pacientes atendidos e "tratados" permanecem sem orientação adequada, vivendo por muitos anos em condição de "cegueira desnecessária". Em nosso país programas de atendimento a portadores de deficiência visual são geralmente concentrados em departamentos de oftalmologia de hospitais universitários. Entretanto, a população deficiente visual deveria ser melhor distribuída nos três níveis de atenção à saúde ao invés de se concentrar o atendimento nos hospitais universitários, já muito sobrecarregados ${ }^{(5)}$. O presente estudo, foi realizado na cidade de Assis fora de uma instituição universitária após a criação da SRDV e trabalho voluntário de oftalmologistas que não só examinaram, diagnosticaram e prescreveram, mas também lideraram movimento para atendimento multidisciplinar e para obtenção dos recursos necessários para compra de auxílios ópticos e não ópticos. Analisando os deficientes visuais em Assis, encontrou-se predomínio de brancos, do sexo masculino e jovens. Esta caracterização amostral encontra-se próxima de outros estudos nacionais referentes à deficiência visual ${ }^{(6-10)}$ particularmente quanto à idade. Quanto ao gênero há controvérsias, alguns tendo encontrado predomínio do sexo feminino ou equilíbrio entre ambos os $\operatorname{sexos}^{(6-8,10)}$.

A maioria dos pacientes apresentou baixa visão estando de acordo com a literatura nacional ${ }^{(6-13)}$ e estrangeira ${ }^{(14-18)}$ que refere que o número de cegos é de 3 a 7 vezes menor que o de portadores de baixa visão.

As causas mais frequentes de deficiência visual no presente estudo em ordem decrescente foram retinocoroidite por toxo- plasmose, atrofia óptica congênita, alta hipermetropia, alta miopia/retinose pigmentar e glaucoma congênito/catarata congênita. A retinocoroidite por toxoplasmose é indicada como a principal causa de deficiência visual em todos os estudos nacionais consultados, variando de 16,7 a $43,5 \%{ }^{(5-13)}$. A atrofia óptica também está entre as principais causas variando de 8,2 a $21,4 \%^{(5-12)}$. As demais causas variam muito entre os diferentes estudos. A retinocoroidite por toxoplasmose não foi observada entre as principais causas de deficiência visual em outros países exceto na Nigéria, com uma frequência de 4,22\% ${ }^{(14)}$. Em outros países aparece também a atrofia óptica ${ }^{(14-19)}$ com frequências variando de 12,2 a $19,1 \%$, portanto muito semelhante a encontrada no presente estudo. O glaucoma congênito também aparece como uma causa importante de cegueira ou baixa visão em todo o mundo, com frequências variando de 6 a $22 \%$ e a retinose pigmentar de 7 e $26 \%^{(14-17,20)}$. Na literatura internacional a catarata (congênita ou não congênita) e cicatrizes corneanas ${ }^{(14-17)}$ aparecem como importantes causas, mas apenas a catarata congênita foi relevante no presente estudo. A retinopatia da prematuridade é apontada em alguns estudos ${ }^{(21)}$ como das cinco principais causas de cegueira, junto com glaucoma congênito e rubéola congênita mas não o foi no presente estudo em nenhum dos dois períodos avaliados $\left(3,4 \%\right.$ no $1^{\circ}$ período e $3,3 \%$ no $2^{\circ}$ período). Provavelmente isto decorre do fato que na cidade de Assis há atendimento oftalmológico aos recém-nascidos prematuros na unidade de terapia intensiva neonatal desde 1994 realizado no Hospital Regional de Assis que atende à grande parte da população. Causas importantes de deficiência visual no adulto como a degeneração macular relacionada à idade não foram encontradas no presente estudo porque a quase totalidade dos pacientes se encontrava nas faixas etárias mais jovens. 
Tabela 3. Tipo de deficiência (cegueira ou baixa visão) e doenças causadoras da deficiência dos deficientes visuais que frequentaram a SRDV e dos deficientes visuais empregados (Assis - SP, 1984 - 2009)

\begin{tabular}{|c|c|c|c|c|c|c|c|c|c|}
\hline & & \multicolumn{4}{|c|}{ Amostra total } & \multicolumn{4}{|c|}{ Pacientes empregados } \\
\hline & & \multicolumn{2}{|c|}{ G1 } & \multicolumn{2}{|c|}{ G2 } & \multicolumn{2}{|c|}{ G1 } & \multicolumn{2}{|c|}{ G2 } \\
\hline & & $\mathbf{n}$ & $(\%)$ & $\mathbf{n}$ & $(\%)$ & $\bar{n}$ & $(\%)$ & $\mathbf{n}$ & $(\%)$ \\
\hline Tipo de & Cego & 17 & 29,3 & 37 & 40,7 & 7 & 41,2 & 4 & 50,0 \\
\hline \multirow[t]{3}{*}{ deficicência } & Baixa visão & 41 & 70,7 & 54 & 59,3 & 10 & 58,8 & 4 & 50,0 \\
\hline & Total & 58 & 100,0 & 91 & 100,0 & 17 & 100,0 & 8 & 100,0 \\
\hline & & \multicolumn{4}{|c|}{$p=0,218^{*}$} & \multicolumn{4}{|c|}{$p=0,986^{*}$} \\
\hline \multirow[t]{30}{*}{ Doenças } & Toxoplasmose congênita & 14 & 24,2 & 18 & 19,8 & 1 & 5,9 & 2 & 25,0 \\
\hline & Atrofia óptica congênita & 10 & 17,3 & 10 & 10,9 & 3 & 17,5 & 0 & 0 \\
\hline & Glaucoma congênito & 5 & 8,6 & 4 & 4,4 & 5 & 29,4 & 0 & 0 \\
\hline & Retinose pigmentar & 5 & 8,6 & 5 & 5,5 & 2 & 11,8 & 3 & 37,5 \\
\hline & Alta hipermetropia & 4 & 6,9 & 9 & 9,9 & 0 & 0 & 0 & 0 \\
\hline & Miopia & 3 & 5,2 & 7 & 7,7 & 1 & 5,9 & 0 & 0 \\
\hline & Catarata congênita & 3 & 5,2 & 6 & 6,6 & 2 & 11,8 & 2 & 25,0 \\
\hline & Microftalmia & 3 & 5,2 & 2 & 2,2 & 0 & 0 & 0 & 0 \\
\hline & Descolamento de retina & 3 & 5,2 & 1 & 1,1 & 0 & 0 & 1 & 12,5 \\
\hline & Retinopatia da prematuridade & 2 & 3,4 & 3 & 3,3 & 0 & 0 & 0 & 0 \\
\hline & Coloboma íris e coróide & 2 & 3,4 & 1 & 1,1 & 1 & 5,9 & 0 & 0 \\
\hline & Ectopia do cristalino & 2 & 3,4 & 1 & 1,1 & 1 & 5,9 & 0 & 0 \\
\hline & Retinopatia diabética & 1 & 1,7 & 0 & 0 & 1 & 5,9 & 0 & 0 \\
\hline & Atrofia macular & 1 & 1,7 & 0 & 0 & 0 & 0 & 0 & 0 \\
\hline & Nistagmos & 0 & 0 & 4 & 4,4 & 0 & 0 & 0 & 0 \\
\hline & Ceratocone & 0 & 0 & 3 & 3,3 & 0 & 0 & 0 & 0 \\
\hline & Glaucoma ângulo estreito & 0 & 0 & 3 & 3,3 & 0 & 0 & 0 & 0 \\
\hline & Estrabismo & 0 & 0 & 2 & 2,2 & 0 & 0 & 0 & 0 \\
\hline & Glaucoma neovascular & 0 & 0 & 2 & 2,2 & 0 & 0 & 0 & 0 \\
\hline & Sarcoidose hipofisária & 0 & 0 & 2 & 2,2 & 0 & 0 & 0 & 0 \\
\hline & Adenoma hipófise & 0 & 0 & 1 & 1,1 & 0 & 0 & 0 & 0 \\
\hline & Catarata complicada & 0 & 0 & 1 & 1,1 & 0 & 0 & 0 & 0 \\
\hline & Displasia septo-óptica & 0 & 0 & 1 & 1,1 & 0 & 0 & 0 & 0 \\
\hline & Hemangioma cerebral & 0 & 0 & 1 & 1,1 & 0 & 0 & 0 & 0 \\
\hline & Síndrome de Jacobson & 0 & 0 & 1 & 1,1 & 0 & 0 & 0 & 0 \\
\hline & Trauma perfurante & 0 & 0 & 1 & 1,1 & 0 & 0 & 0 & 0 \\
\hline & Vogt-Koyanagi-Harada & 0 & 0 & 1 & 1,1 & 0 & 0 & 0 & 0 \\
\hline & Atrofia óptica traumática & 0 & 0 & 1 & 1,1 & 0 & 0 & 0 & 0 \\
\hline & Total & 58 & 100,0 & 91 & 100,0 & 17 & 100,0 & 8 & 100,0 \\
\hline & & \multicolumn{4}{|c|}{$\mathrm{p}=0,828^{* *}$} & \multicolumn{4}{|c|}{$p=0,088$} \\
\hline
\end{tabular}

SRDV= Sala de Recursos para Deficientes Visual; G1= grupo 1 (de 1984 a 1996); G2= grupo 2 (de 1997 a 2009)

Nota: As doenças estão na ordem decrescente do $\mathrm{G} 1$

$\left({ }^{*}\right)=$ teste de Qui-quadrado; $\left({ }^{*}\right)=$ teste exato de Fisher

O resultado deste estudo aponta que a principal causa de cegueira ou baixa visão em nosso meio continua sendo a toxoplasmose ocular, que é, uma doença prevenível e portanto mostra a necessidade de implementação de medidas preventivas para evitá-la.

Foi prescrito auxílio óptico ou não óptico para a grande maioria dos pacientes sendo que os mesmos foram efetivamente utilizados pois os oftalmologistas estimularam diversas entidades que os forneceram. Este trabalho dos oftalmologistas foi fundamental, pois no estado de São Paulo foi observado que dos $85,7 \%$ auxílios prescritos apenas $58,3 \%$ foram adquiridos ${ }^{(22)}$.

A análise dos deficientes visuais empregados mostrou características demográficas semelhantes entre os dois grupos quanto ao gênero, raça/cor, observando-se no entanto maior frequência de pacientes nas faixas etárias acima de 20 anos em ambos. Também houve predomínio de portadores de baixa visão, mas aumentou a porcentagem de cegos em relação à amostra total. Este achado mostra que mesmo pacientes considerados legalmente cegos, quando encontram condições de atendimento oftalmológico e educação adequados podem se inserir no mercado de trabalho.

A frequência das doenças dos pacientes empregados foi diferente das da amostra total. As duas doenças mais frequentes nos pacientes empregados foram o glaucoma congênito e atrofia óptica, em G1 e a retinose pigmentar e catarata congênita em G2, mas pelo pequeno número de cada uma delas não podemos concluir que estas doenças poderiam ser um fator de facilitação para o emprego. 


\begin{tabular}{|c|c|c|c|}
\hline & \multicolumn{3}{|c|}{ Empregados } \\
\hline & \multicolumn{2}{|c|}{ Sim } & \multirow[t]{2}{*}{$\mathbf{p}$} \\
\hline & G1 & G2 & \\
\hline Gênero M (\%) & 58,8 & 25,0 & $0,201\left(^{*}\right)$ \\
\hline Idade (anos) & $28,0(19,0 ; 50,0)$ & $30(25 ; 34,5)$ & $0,0726\left(^{* *}\right)$ \\
\hline Brancos (\%) & 82,4 & 62,5 & $0,330\left(^{*}\right)$ \\
\hline Baixa visão (\%) & 58,8 & 50,0 & $0,149\left(^{\star \star}\right)$ \\
\hline \multicolumn{4}{|l|}{ Doenças (\%) } \\
\hline Toxoplasmose & 5,9 & 25,0 & \multirow{5}{*}{$\mathrm{p}=0,119\left(^{*}\right)$} \\
\hline Atrofia & 17,6 & 0,0 & \\
\hline Glaucoma & 29,4 & 0,0 & \\
\hline Retinose & 11,8 & 37,5 & \\
\hline Outros & 35,3 & 37,5 & \\
\hline
\end{tabular}

O estudo mostrou boa frequência à SRDV e uso de recursos ópticos em mais de 50\% dos pacientes empregados, sugerindo que estes dois fatores favoreceram a empregabilidade, pois permitiram a participação dos pacientes em ambientes acadêmicos competitivos ${ }^{(23)}$ apesar da análise de associação não ter sido significativa.

A análise de associação entre emprego e os diferentes parâmetros só mostrou nível de significância para a idade, sendo a mediana da idade dos pacientes empregados 10 anos maior do que a dos não empregados, sugerindo que com a continuidade de atendimento oftalmológico e educação adequadas os pacientes mais jovens poderão se empregar, o que aumentaria ainda mais a taxa de emprego que neste trabalho atingiu 44,7\% no G1. A falta de significância de associação de emprego com aspectos que se esperariam importantes como: frequência à SRDV, nível de escolaridade e tipo de deficiência poderia ser explicada pela amostragem pequena. A redução importante da taxa de emprego no segundo período para $12,3 \%$ foi um resultado inesperado ainda mais que a escolaridade foi maior neste grupo. Analisando o número de pacientes aposentados nos dois grupos foi encontrado aumento do número de aposentados em G2 que foi de 38,46\%, quando G1 era de apenas 7,9\%. Isto sugere que parte dos pacientes de $\mathrm{G} 2$ que poderiam estar trabalhando está usufruindo dos benefícios da previdência social garantidos pelo decreto $\mathrm{n}^{\circ} 3.298$ de 1999 .

Não foi encontrado na literatura nacional estudos sobre taxa de emprego dos deficientes visuais o que dificulta comparações. A taxa de emprego de deficientes visuais nos Estados Unidos é muito menor do que a de não deficientes visuais que é $82 \%{ }^{(24)}$. A taxa de emprego de pacientes cegos com idade de 21 a 64 anos é de $31 \%$ e de portadores de baixa visão é de $44 \%{ }^{(25)}$.

\section{CONCLUSÃO}

Assim a taxa de emprego dos deficientes visuais na cidade de Assis durante o período de 1984 a 1996 se assemelha a de um país desenvolvido, mostrando que a atuação conjunta de oftalmologistas, educadores e da sociedade podem facilitar a inclusão social do deficiente visual. No entanto esforços devem continuar para manter a taxa de emprego nestes níveis, melhorando e facilitando as condições de empregabilidade.

\section{AGRADECIMENTO}

Os autores agradecem o Sr. Hélio Rubens de Carvalho Nunes CONRE 8480A pela análise estatística.

\section{ABSTRACT}

Purposes: To establish the visually impaired resource class users profile in the city of Assis - SP, to estimate the visually impaired employment percentage and to compare employment rate with age, gender, deficiency type and causing disease between two periods:1984 - 1996 and 1997 - 2009. Methods: Retrospective review of both medical and school records from visually impaired individuals attending visually impaired resource class in Assis - SP between 1984 and 2009. This time period was divided into two intervals: 1984 - 1996 (G1) and 1997 - 2009 (G2). Collected data included demographic features and diseases, type of visual impairment (blindness or low vision), education, optic aids, attendance to visually impaired resource class and employment rate. Employment rate was associated with age, gender, type of visual impaiment and causing disease between both study time intervals. Results: A total of 149 visually impaired individuals were identified - $61.07 \%$ male, $38.9 \%$ female, $82.5 \%$ white, and $17.4 \%$ non-white. Mean age was 18 years. Of the 149 visually impaired, $63.75 \%$ had low vision and $36.24 \%$ were blind. In decreasing order, the more frequent conditions leading to visual impairment were toxoplasmosis retinochoroiditis (17.40\%), congenital optic atrophy (9.39\%), high hyperopia $(8.72 \%)$, pigmentary retinosis and high myopia (with $6.71 \%$ each) and congenital glaucoma and congenital cataract (with $6.04 \%$ each). Attendance to visually impaired resource class was good by over $50 \%$ of the patients. Of the individuals $>14$ years-old, $44.7 \%$ and $12.3 \%$ were regularly employed during 1984 - 1996 and $1997-2009$ periods, respectively. Employment rate only correlated with age (individuals with higher mean age were most frequently employed) and there was not correlation with demographic characteristics, deficiency type and causing diseases. Conclusions: The main characteristics of the individuals attending the visually impaired resource class are: male, white, with low vision and ocular toxoplasmosis. Nearly half of the individuals older than 14 years-old $(44.7 \%)$ were regularly employed up to 1996, suggesting that the joint effort of ophthalmologists and teachers helps the social inclusion of these patients. Employment rate dropped over 1997 - 2009 period, and the rate of retirees increased. 
Keywords: Vision disorders; Blindness; Vision, low/rehabilitation; Employment; Community health centers; Socialization

\section{REFERÊNCIAS}

1. Rocha H, Ribeiro-Gonçalves E. Ensaios sobre a problemática de cegueira. Belo Horizonte: Ed. Fundação Hilton Rocha; 1987. p.354.

2. Brasil. Ministério da Educação. Secretaria Estadual de Educação. Resolução 95/ 2000, de 21 de novembro de 2000. Dispõe sobre o atendimento dos alunos com necessidades educacionais da rede estadual de São Paulo. Brasília: MEC: 2000.

3. Carvalho KMM, Freitas CC de, Kimoto EM, Gasparetto MERF. Avaliação e conduta em escolares portadores de visão subnormal atendidas em sala de recursos. Arq Bras Oftalmol. 2002;65(4):445-9.

4. Pinto ALT, Windt MCV, Céspedes L. Vade Mécum. São Paulo: Saraiva; 2000. Consolidação das Leis do Trabalho.

5. Kara-José N, Almeida GV de, Arieta CEL, Araújo JS, Bechara SJ, Oliveira PR. Causas de deficiência visual em crianças. Bol Oficina Sanit Panam. 1984;97(5): 405-13.

6. Alves LS. Causas de cegueira e visão subnormal no Centro Louis Braille-Porto Alegre. Arq Bras Oftalmol. 1985;48(2):65-7.

7. Kara-José N, Carvalho KMM, Pereira VL, Venturini NHB, Gasparetto MEFR, Gushikem MT. Estudo Retrospectivo dos Primeiros 140 casos Atendidos na Clinica de Visão Subnormal do Hospital de Clinicas da UNICAMP. Arq Bras Oftalmol. 1988;51(2):65-9.

8. Buchignani BPC, Silva MRBM. Serviço de visão subnormal do Hospital das Clinicas de Botucatu: levantamento de causas e resultados. Arq Bras Oftalmol. 1991;50(5):305-10.

9. Moreira ATR, Moreira Jr CA, Arana J. Causas de cegueira no Instituto de Cegos do Paraná. Arq Bras Oftalmol. 1991;54(6):275-8.

10. Leal DB, Tavares SS, Ventura LO, Florêncio T. Atendimento a portadores de visão subnormal. estudo retrospectivo de 317 casos. Arq Bras Oftalmol. 1995; 58(6):439-42.

11. Barbieri LC. Atendimento de escolares e pré-escolares com visão subnormal. Arq Bras Oftalmol. 1984;47(3):107-10.
12. Tartarella MB, Nakano K, Castro CTM, Martins APM. Visão subnormal em crianças. Arq Bras Oftalmol. 1991;54(5):221-4.

13. Esteves JF, Domingues CG, Borges LPK, Skolaude PBV, Bortolomiol L, Muxfeldt RA, et al. Prevalência e causas de cegueira em Bairro de Porto Alegre. Arq Bras Oftalmol. 1996;59(3):244-7.

14. Olurin O. Etiology of blindness in Nigerian children. Am J Ophtalmol. 1970; 70(4):533-40

15. Baghdassarian SA, Tabbara KF. Childhood blindness in Lebanon. Am J Ophtalmol. 1975;79(5):827-30.

16. Phillips CI, Levy AM, Newton M, Stokoe NL. Blindness in schoolchildren: importance of heredity, congenital cataract, and prematurity. Brit J Ophtalmol. 1987;71(8):578-84.

17. Jay B. Causes of blindness in schoolchildren. Br Med J (Clin Res Ed). 1987; 294(6581):1183-4.

18. Goggin M, O‘Keefe M. Childhood blindness in the Republic of Ireland: a national survey. Bri J Opthalmol. 1991;75(7):425-9.

19. Elder MJ, De Cock R. Childhood blindness in the West Bank and Gaza Strip: prevalence, aetiology and hereditary factors. Eye (Lond). 1993;7(Pt 4):580-3.

20. Bucher PJ, Ijsselmuiden CB. Prevalence and causes of blindness in the Northern Transvaal. Br J Ophtalmol. 1988;72(10):721-6.

21. Brito PR, Veitzman S. Causas de cegueira e baixa visão em crianças. Arq Bras Oftalmol. 2000;63(1):49-54.

22. Monteiro GB, Temporini ER, Carvalho KM. Use of optical aids by visually impaired students: social and cultural factors. Arq Bras Oftalmol. 2006;69(4): 503-7.

23. Apushkin MA, Fishman GA Attainment of educational level in patients with Leber congenital amaurosis. Ophthalmololgy. 2006;113(3):481-2.

24. Dixon K, Kruse D, Van Horn C. Work trends: Americans' attitudes about work, employers and government. Restricted acess: A survey of imployers about people with disabilities and lowering barriers to work [Internet]. New Brunswich, NJ. Heldrich Center for Workforce Development; 2003. p.7. [cited 2009 Jan 25]. Available from:http://www.heldrich.rutgers.edu/uploadedFiles/Publications/ WT16\%20Press\%20Release.pdf

25. Wolffe K, Candela A. Expanding the labor pool: recruiting, hiring, and maintaining workers with visual impairments. Employment Relations Today. 2002; 29:59-68 